\title{
Synchronous, Nonsynchronous and Negative Rotations: How Spin and Gravity Orchestrate Planetary Motions
}

\author{
Puthalath Koroth Raghuprasad ${ }^{1}$ \\ ${ }^{1} 2400$ E. $8^{\text {th }}$ Street, Odessa, Texas, United States of America \\ Correspondence: Puthalath Koroth Raghuprasad, 2400 E. $8^{\text {th }}$ Street, Odessa 79761, Texas, United States of \\ America. Tel: 432-349-6566. E-mail: pkrp12@gmail.com
}

Received: October 13, 2019

Accepted: November 272019

Online Published: January 31, 2020

doi:10.5539/apr.v12n2p1

URL: http://dx.doi.org/10.5539/apr.v12n2p1

\begin{abstract}
This study identifies the unique features accompanying the phenomenon of synchronous rotation of the major (proximal) satellites of the gas giants and the earth's moon, and the special features leading to the 'negative' rotation of Venus, Uranus and Pluto, as well as the most peripheral small satellites of the gas giants. Such features help us understand how these phenomena occur but also, by combining all of the observations help explain other (regular) planetary motions as well. In the synchronously rotating satellites, the salient features are the satellites' low axial tilts and both the orbital speed and the axial rotation speed increasing with proximity to the mother body. In "negative" rotation, axial tilts are in excess of $120^{\circ}$ and the axial rotation speeds are significantly delayed; this delay is most pronounced in Venus, which has an axial tilt of $-174^{\circ}$. A scrutiny of the orbital parameters of all the satellites of the gas giants alone will yield sufficient data to propose a working hypothesis of how mutual gravitation, combined with spin (axial rotation and orbital motion), the distance from the mother, and centrifugal force can explain all motions. It confirms our belief that the process of planetary motions is a continuum from the synchronous, through degrees of non-synchronicity (or regular orbits), to the negative rotations, all depending on the degree of influence from mother bodies, as a product of distances from them. Thus, the nearest large satellites with the least axial tilts display synchronous rotation. Those satellites that are intermediate in distance from the mother show nonsynchronous axial rotation and correspondingly slower orbital speeds. The small peripheral satellites display axial tilts over 120 degrees and rotate negatively. In all these orbital motions, centrifugal force is the crucial restraining influence; lest, the orbiting bodies will tend to fall into the mother bodies. How all these pieces of the puzzle fit together in the orderly movements of bodies in the universe is the underlying theme of this article.
\end{abstract}

Keywords: Spin, Orbit, Axial Rotation, Gravity, Solar System, Gas Giants

\section{Introduction}

The "synchronous rotation" (the orbital period of the satellites is the same as their axial rotation periods) is a phenomenon that is displayed by the closest major satellites of the gas giants, and by our moon. When the distance between the mother and daughter is right, synchronicity of the orbit exists and only one face of the satellite is visible. Those satellites that are located farther away do not display this ability and their orbits are nonsynchronous. Scientists explain the synchronous rotation as being due to a "tidal locking" mechanism (Murray, Dermott, \& Stanley, 1999; Goldman, Quinn, Nicholson, \& Rand, n. d.). However, since the satellites are also rotating on their axes, a simple tidal locking cannot explain the synchronicity. Tidal locking can only explain the increase in the orbital speed the closer the satellites are to the mother body. The increase in the speed of rotation (which is in addition to the increased speed of orbit) of the closest satellites, which is presented in this paper, cannot be explained by tidal locking alone. Since the satellite bodies are also rotating on their axes and yet only the same face is visible, a simple 'tidal locking' mechanism also fails to explain this finding associated with synchronous rotation. Rather, direct 'rotational' effect from the mother to the satellite(s), coupled with gravity and centrifugal force will explain all the observed phenomena. Thus, the closer the satellites, the faster the orbital speed and equally faster the axial rotation. One focus of this paper is to explain how such effects are transmitted by the mother to the satellites.

This paper also explores the other curious finding in the orbital parameters of the planets, the 'negative rotation' ('reverse rotation', the axial rotation being opposite in direction to those of the Sun and most of the planets). In 
the phenomenon of negative rotation, excessive axial tilt plays a pivotal role; the way in which this excessive axial tilt leads to the delay in their axial rotation speeds is explained later in this paper.

The sum total of all the findings reported in this paper support the notion that spin is a purposeful property of freestanding celestial bodies. It is further proposed that even the orderly orbits of the planets around the Sun and the satellites around their mother planets, involve the combined forces of mutual gravitation (which obeys the "Inverse square law" (Seeds, 1999)) but requires this spin/rotational influence from the mother bodies to guide the direction of such orbits. They also help explain how, the complementary nature of the axial rotation and gravity can explain many other observed phenomena in our solar system and in the larger universe. Therefore, when appropriate, reasonable extrapolations will be made to explain some other observed phenomena in the solar system, which are directly influenced by spin and gravity and about their complementary roles in the universe as well.

\section{Materials and Observations}

As the solar system can be considered a representative unit of the universe, with a complex system of bodies obeying roughly the same physics that perhaps exists in all parts of the universe, the available data about that system was reviewed. This decision was also influenced by the fact that such data are available in sufficient detail for our purposes. Thus, the data dealing with the solar system, provided by NASA on their website (http://nssdc.gsfc.nasa.gov.html) were studied in depth. Close attention was paid to the rotational and orbital parameters and those other factors that might have a bearing on them. The distance from the mother body, the mass and size of each body, and the axial tilts, gravity and other relevant properties were examined in detail. Current astronomical literature referenced in this paper was also studied as it applied to the architectural and functional aspects of the universe. The results of our review of the literature and the data on NASA's website are presented in the tables and figures as described below.

Table 1. Selected planetary facts sheet

\begin{tabular}{|c|c|c|c|c|c|c|c|c|c|}
\hline & Mercury & Venus & Earth & Mars & Jupiter & Saturn & Uranus & Neptune & Pluto \\
\hline $\begin{array}{l}\text { Mass } \\
\left(10^{24} \mathrm{Kg}\right)\end{array}$ & 0.330 & 4.87 & 5.97 & 0.642 & 1,899 & 568 & 86.8 & 102 & 0.0125 \\
\hline $\begin{array}{l}\text { Diameter } \\
(\mathrm{Km})\end{array}$ & 4,879 & 12,104 & 12,756 & 6,792 & 142,984 & 120,536 & 57,118 & 49,528 & 2,390 \\
\hline $\begin{array}{l}\text { Density } \\
\left(\mathrm{Kg} / \mathrm{m}^{3}\right)\end{array}$ & 5,427 & 5,243 & 5,515 & 3,933 & 1,326 & 687 & 1,270 & 1,638 & 1,750 \\
\hline $\begin{array}{l}\text { Distance From sun } \\
\left(10^{6} \mathrm{Km}\right)\end{array}$ & 57.9 & 108.2 & 149.6 & 227.9 & 778.6 & $1,433.5$ & $2,872.5$ & $4,495.1$ & 5,870 \\
\hline $\begin{array}{l}\text { Orbital Velocity } \\
(\mathrm{Km} / \mathrm{sec})\end{array}$ & 47.9 & 35 & 29.8 & 24.1 & 13.1 & 9.7 & 6.8 & 5.4 & 4.7 \\
\hline $\begin{array}{l}\text { Orbital Period } \\
\text { (Days) }\end{array}$ & 88 & 224.7 & 365.2 & 687 & 4,331 & 10,747 & 30,589 & 59,800 & 90,588 \\
\hline $\begin{array}{l}\text { Perihelion } \\
\left(10^{6} \mathrm{Km}\right)\end{array}$ & 46 & 107.5 & 147.1 & 206.6 & 740.5 & $1,352.6$ & $2,741.3$ & $4,444.5$ & 4,435 \\
\hline $\begin{array}{l}\text { Aphelion } \\
\left(10^{6} \mathrm{Km}\right)\end{array}$ & 69.8 & 108.9 & 152.1 & 249.2 & 816.6 & $1,514.5$ & $3,003.6$ & $4,545.7$ & $7,304.3$ \\
\hline $\begin{array}{l}\text { Gravity } \\
\left(\mathrm{M} / \mathrm{s}^{2}\right)\end{array}$ & 3.7 & 8.9 & 9.8 & 3.7 & 23.1 & 9 & 8.7 & 11 & 0.6 \\
\hline $\begin{array}{l}\text { Axial Tilt } \\
\text { (Degrees) }\end{array}$ & 0.01 & 177.4 & 23.4 & 25.2 & 3.1 & 26.7 & 97.8 & 28.3 & 122.5 \\
\hline $\begin{array}{l}\text { Rotation } \\
\text { Period (h) }\end{array}$ & $1,407.6$ & $*_{-5,832.5}$ & 23.9 & 24.6 & 9.9 & 10.7 & $*_{-17.2}$ & 16.1 & $*-153.3$ \\
\hline
\end{tabular}

*Negative rotation $=$ axial rotation opposite to that of the sun's

Adapted from: http://nssdc.gsfc.nasa.gov/planetary/factsheet/index.html 
Table 2. Orbital Parameters of Satellites of Jupiter*

\begin{tabular}{|c|c|c|c|c|c|}
\hline Satellites: & $\begin{array}{l}\text { Radius } \\
(\mathrm{Km})\end{array}$ & $\begin{array}{l}\text { Distance from Jupiter }{ }^{\Delta} \\
\left(10^{3} \mathrm{Km}\right)\end{array}$ & $\begin{array}{l}\text { Orbital Period } \\
\text { (Days) } \\
\end{array}$ & $\begin{array}{l}\text { Rotation Period } \\
\text { (Days) }\end{array}$ & $\begin{array}{l}\text { Inclination } \\
\text { (Degrees) }\end{array}$ \\
\hline \multicolumn{6}{|l|}{ A) Galilean: } \\
\hline Io & $1,821.6$ & 421.6 & 1.769138 & $\mathrm{~S}$ & 0.04 \\
\hline Europa & $1,560.8$ & 670.9 & 3.551181 & $\mathrm{~S}$ & 0.47 \\
\hline Ganymede & $2,631.2$ & $1,070.4$ & 7.154553 & $\mathrm{~S}$ & 0.21 \\
\hline Callisto & $2,410.3$ & $1,882.7$ & 16.689018 & $\mathrm{~S}$ & 0.51 \\
\hline \multicolumn{6}{|l|}{ B) 'Lesser' } \\
\hline Metis & 20 & 128 & 0.294779 & $\mathrm{~S}$ & 0.06 \\
\hline Adrastea & $13 \times 10 \times 8$ & 129 & 0.298260 & $\mathrm{~S}$ & 0.03 \\
\hline Amalthea & $131 \times 73 \times 67$ & 181.4 & 0.498179 & $\mathrm{~S}$ & 0.40 \\
\hline Thebe & $55 \times 45$ & 221.9 & 0.6745 & ND & 0.8 \\
\hline Themisto & 4 & 7,507 & 132.02 & ND & 45.67 \\
\hline Leda & 5 & 11,170 & 240.92 & ND & 27.47 \\
\hline Himalia & 85 & 11,460 & 250.5662 & 0.4 & 27.63 \\
\hline Lysithea & 12 & 11,720 & 259.22 & ND & 27.35 \\
\hline \multirow{2}{*}{$\begin{array}{l}\text { Elara } \\
\text { S/2000 J11 }\end{array}$} & 40 & 11,740 & 259.6528 & 0.5 & 24.77 \\
\hline & 2.0 & 12,560 & 287.0 & ND & 28.2 \\
\hline $\begin{array}{l}\text { Carpo } \quad(\mathrm{S} / 2003 \\
\mathrm{J} 20)\end{array}$ & 3.0 & 16,990 & 456.1 & ND & 51.4 \\
\hline Euporie & 1 & 19,390 & $553.1 \mathrm{R}$ & $\mathrm{ND}$ & 147.0 \\
\hline Orthosie & 1 & 20,720 & $622.6 \mathrm{R}$ & $\mathrm{ND}$ & 145.9 \\
\hline Euanthe & 1.5 & 20,800 & $620.6 \mathrm{R}$ & $\mathrm{ND}$ & 148.9 \\
\hline Thyone & 2 & 20,940 & $627.3 \mathrm{R}$ & $\mathrm{ND}$ & 148.5 \\
\hline Mneme & 2.0 & 21,070 & $620.0 \mathrm{R}$ & ND & 148.6 \\
\hline Harpalyke & 2.2 & 21,110 & $623.3 \mathrm{R}$ & $\mathrm{ND}$ & 148.7 \\
\hline Hermippe & 2 & 21,130 & $633.9 \mathrm{R}$ & $\mathrm{ND}$ & 150.7 \\
\hline Praxidike & 3.4 & 21,150 & $625.3 \mathrm{R}$ & ND & 148.7 \\
\hline Thelxinoe & 2.0 & 21,160 & $628.1 \mathrm{R}$ & $\mathrm{ND}$ & 151.4 \\
\hline Helike & 4.0 & 21,260 & $634.8 \mathrm{R}$ & $\mathrm{ND}$ & 154.8 \\
\hline Iocaste & 2.6 & 21,270 & $631.5 \mathrm{R}$ & ND & 159.7 \\
\hline Ananke & 10 & 21,280 & $629.8 \mathrm{R}$ & $\mathrm{ND}$ & 148.9 \\
\hline Eurydome & 1.5 & 22,870 & $717.3 \mathrm{R}$ & ND & 150.3 \\
\hline Arche & 1.5 & 22,930 & $723.9 \mathrm{R}$ & ND & 165.0 \\
\hline Autonoe & 2 & 23,040 & $762.7 \mathrm{R}$ & $\mathrm{ND}$ & 152.9 \\
\hline Herse & 2.0 & 23,097 & $715.4 \mathrm{R}$ & $\mathrm{ND}$ & 164.2 \\
\hline Pasithee & 1 & 23,100 & $716.3 \mathrm{R}$ & ND & 165.4 \\
\hline Chaldene & 1.9 & 23,180 & $723.8 \mathrm{R}$ & $\mathrm{ND}$ & 165.4 \\
\hline Kale & 1 & 23,220 & $729.5 \mathrm{R}$ & ND & 165.0 \\
\hline Isonoe & 1.9 & 23,220 & $725.5 \mathrm{R}$ & ND & 165.0 \\
\hline Aitne & 1.5 & 23,230 & $730.2 \mathrm{R}$ & $\mathrm{ND}$ & 165.1 \\
\hline Erinome & 1.6 & 23,280 & $728.3 \mathrm{R}$ & ND & 164.9 \\
\hline Taygete & 2.5 & 23,360 & $732.2 \mathrm{R}$ & ND & 165.2 \\
\hline Carme & 15 & 23,400 & $734.2 \mathrm{R}$ & $\mathrm{ND}$ & 164.9 \\
\hline Sponde & 1 & 23,490 & $748.3 \mathrm{R}$ & ND & 151 \\
\hline Kalyke & 2.6 & 23,580 & $743 \mathrm{R}$ & ND & 165.2 \\
\hline Pasiphae & 18 & 23,620 & $743.6 \mathrm{R}$ & $\mathrm{ND}$ & 151.4 \\
\hline Eukelade & 4.0 & 23,660 & $746.4 \mathrm{R}$ & $\mathrm{ND}$ & 165.5 \\
\hline Megaclite & 2.7 & 23,810 & $752.8 \mathrm{R}$ & $\mathrm{ND}$ & 152.8 \\
\hline Sinope & 14 & 23,940 & $758.9 \mathrm{R}$ & $\mathrm{ND}$ & 158.1 \\
\hline Hegemono & 3.0 & 23,950 & $739.6 \mathrm{R}$ & $\mathrm{ND}$ & 155.2 \\
\hline Aoede & 4.0 & 23,980 & $761.5 \mathrm{R}$ & ND & 158.3 \\
\hline Kallichore & 2.0 & 24,040 & $764.7 \mathrm{R}$ & $\mathrm{ND}$ & 165.5 \\
\hline Callirrhoe & 4 & 24,100 & $758.8 \mathrm{R}$ & ND & 147.1 \\
\hline Cyllene & 2.0 & 24,350 & $737.8 \mathrm{R}$ & ND & 149.3 \\
\hline Kore & 2.0 & 24,540 & $779.2 \mathrm{R}$ & ND & 152.4 \\
\hline
\end{tabular}


C) Newly discovered satellites $\mathrm{S} / 2000 \mathrm{~J} 2$ to $\mathrm{S} / 2011 \mathrm{~J} 2$ have orbital periods from 504 to 982.5 ; all exhibit reverse 'motion' and orbital inclination from 140.8 to 165

$\mathrm{S}=$ Synchronous rotation (rotation period is the same as orbital period) $\mathrm{R}=$ Retrograde rotation $\mathrm{ND}=$ No data available

${ }^{\Delta}$ Distance from Jupiter $\left(10^{3} \mathrm{~km}\right)=$ Semi-major Axis

*Adapted from:http://nssdc.gsfc.nasa.gov/planetary/factsheet/joviansatfact.html April 19, 2013

Reproduced with kind permission of Physics Essays Publication, http://physicsessays.org/ with modifications.

Table 3. Synchronous* Rotation in Earth's Moon and the Major Satellites of the Gas Giants^

\begin{tabular}{|c|c|c|c|c|c|c|}
\hline Planet & Satellites & Diameter $(\mathrm{Km})$ & $\begin{array}{l}\text { Distance from Mother }{ }^{\Delta} \\
\left(10^{3} \mathrm{~km}\right)\end{array}$ & $\begin{array}{l}\text { Orbital } \\
\text { Period } \\
\text { (Hours) }\end{array}$ & Rotation + Period & Orbital Velocity $(\mathrm{km} / \mathrm{sec})$ \\
\hline Earth & Moon & $3,476.2$ & 384 & 655.2 & $\mathrm{~S}$ & 1.023 \\
\hline \multirow{4}{*}{ Jupiter } & Io & 3643.2 & 421.6 & 42.456 & 42.5 & 17.3 \\
\hline & Europa & 3121.6 & 670.9 & 85.224 & 85.2 & 13.7 \\
\hline & Ganymede & 5262.4 & 1070.4 & 171.696 & 171.7 & 10.9 \\
\hline & Callisto & 4820.6 & 1882.7 & 400.536 & 400.5 & 8.2 \\
\hline \multirow{8}{*}{ Saturn } & Mimas & $416 \times 394 \times 382$ & 185.52 & 22.618 & $\mathrm{~S}$ & ND \\
\hline & Enceladus & $514 \times 502 \times 496$ & 238.02 & 32.885 & $\mathrm{~S}$ & ND \\
\hline & Tethys & $1076 \times 1056 \times 1052$ & 294.66 & 45.307 & $\mathrm{~S}$ & ND \\
\hline & Dione & $1126 \times 1122 \times 1120$ & 377.40 & 65.686 & $\mathrm{~S}$ & ND \\
\hline & Rhea & $1530 \times 1526 \times 1524$ & 527.04 & 108.42 & $\mathrm{~S}$ & ND \\
\hline & Titan & 5150 & $1,221.83$ & 382.69 & $\mathrm{~S}$ & ND \\
\hline & Hyperion & $360 \times 266 \times 206$ & $1,481.1$ & 510.638 & $\mathrm{~S}$ & ND \\
\hline & Iapetus & $1492 \times 1492 \times 1424$ & $3,561.3$ & 1903.924 & $\mathrm{~S}$ & ND \\
\hline \multirow{5}{*}{ Uranus } & Miranda & $480 \times 468.4 \times 465.8$ & 129.39 & 33.923 & $\mathrm{~S}$ & ND \\
\hline & Ariel & $1162.2 \times 1155.8 \times 1155.4$ & 191.02 & 60.489 & $\mathrm{~S}$ & ND \\
\hline & Umbriel & 1169.4 & 266.30 & 99.46 & $\mathrm{~S}$ & ND \\
\hline & Titania & 1577.8 & 435.91 & 208.94 & $\mathrm{~S}$ & ND \\
\hline & Oberon & 1522.8 & 583.52 & 323.117 & $\mathrm{~S}$ & ND \\
\hline
\end{tabular}

* Synchronous Rotation $(\mathrm{S})=$ Rotation period is the same as the orbital period

${ }^{\wedge}$ Neptune's Satellites are not included as the details about the rotation are unavailable

${ }^{\Delta}$ Distance from Mother $=$ Semimajor Axis

+ Rotation period is available only for Jupiter's satellites. However, since all of the other satellites listed in this table rotate "synchronously," for them the orbital period was used instead, for rotation period.

ND $=$ No Data Available

*Adapted from: http://nssdc.gsfc.nasa.gov/planetary/factsheet

Table 4. Comparison of Planets with Negative Rotation* (Venus, Uranus, and Pluto), to Earth and Jupiter

\begin{tabular}{llllll}
\hline & Venus & Uranus & Pluto & Earth & Jupiter \\
\hline Mass $\left(10^{24} \mathrm{Kg}\right)$ & 4.87 & 86.8 & 0.0125 & 5.97 & 1,899 \\
Diameter $(\mathrm{Km})$ & 12,104 & 51,118 & 2,390 & 12,756 & 142,984 \\
Rotation Period (Hours) & $-5,832.5^{*}$ & $-17.2^{*}$ & $-153.3^{*}$ & 23.9 & 9.9 \\
Length of Day (hrs) & 2,802 & 17.2 & 153.3 & 24 & 9.9 \\
Orbital inclination (Degrees) & 3.4 & 0.8 & 17.2 & 0.0 & 1.3 \\
Axial Tilt (Degrees) & 177.4 & 97.8 & 122.5 & 23.4 & 3.1 \\
Magnetic Field & No & Yes & Unknown & Yes & Yes \\
\hline
\end{tabular}

*Negative rotation means axial rotation opposite to that of the Sun

Adapted from: http://nssdc.gsfc.nasa.gov/planetary/factsheet/index.html

Reproduced with kind permission of Physics Essays Publication, http://physicsessays.org/ 
Table 1 presents the orbital parameters of all the planets of our solar system and some other salient features. Included in the analyses are the mass, diameter, density, distance from the Sun, orbital velocity, orbital period, aphelion, perihelion, gravity, axial tilt and rotation period. The distance from the Sun shows a direct relationship with the orbital velocity, as predicted by the inverse square law. Both perihelion and aphelion also increase with the distance from the Sun. One curious feature is the 'negative' axial rotation in Venus, Uranus and Pluto; both Venus and Pluto also display undue delay in the rotation period. All of them also display large axial tilts (over 90 degrees) and, considerable slowing of the axial rotation in both Venus and Pluto. Explanation of this follows in the Discussion section.

Table 2 presents the orbital parameters of Jupiter and its known satellites. Jupiter's was chosen as a prototypical planetary system but Saturn's and Uranus' systems follow a similar pattern but are not shown here for the sake of brevity (these were reported in ref \#19). Neptune's details are incomplete at present. All of them confirm the accuracy of the inverse square law as it applies to these planets and their satellites as well. This table also demonstrates the 'synchronicity' of the large satellites that are closest to the gas giants and the 'reverse rotation' of the 'lesser' satellites that are farthest. The satellites in intermediate distances from Jupiter, display nonsynchronous rotation.

Table 3 compares the closest major satellites of the gas giants Jupiter, Saturn and Uranus and the earth's own moon. All of them orbit their respective mother planets in a synchronous manner. The axial tilts of these satellites of the gas giants are not available for review but that of our moon is only $6.7^{\circ}$. NASA's website does indicate that the orbital inclination of these bodies is less than $2^{\circ}$, with only an odd satellite displaying orbital inclination higher but most of them are still below $15^{\circ}$. It is not known if the axial tilts will parallel the orbital inclination. It is to be stressed that all of these satellites are situated close to the mother bodies. Thus, a large gravitational influence from the mother body must have a major role in this phenomenon (synchronicity). This phenomenon is further explored below and in the Discussion.
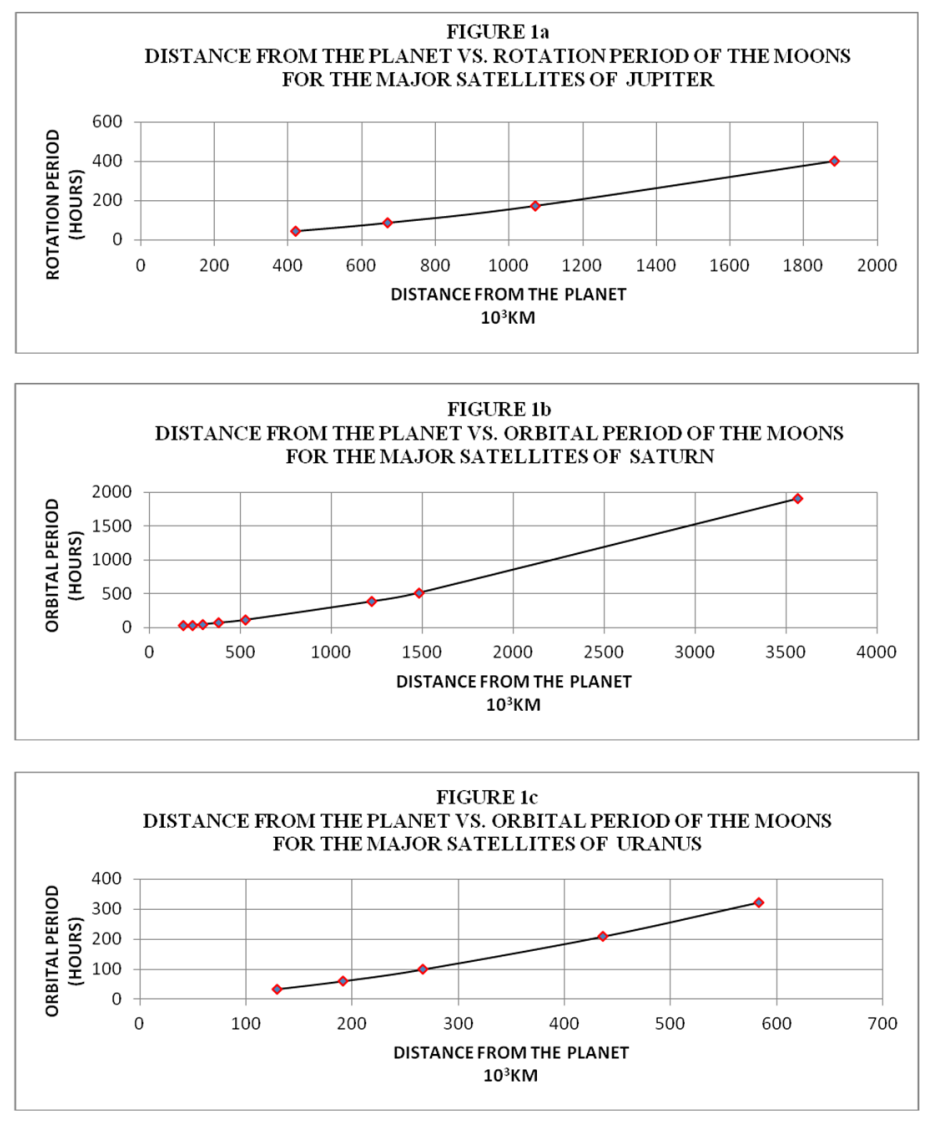

Figure 1

These figures compare the Distance from the Mother Body vs. the Rotation Period (orbital period in Saturn and Uranus) of the Synchronously Rotating Major Satellites of Jupiter, Saturn, and Uranus (from Table 3).

All three comparisons indicate an extremely positive linear relationship $(r=0.9959,0.9893$, and 0.9962 
Respectively).

Adapted from: http://nssdc.gsfc.nasa.gov/planetary/factsheet/index.html

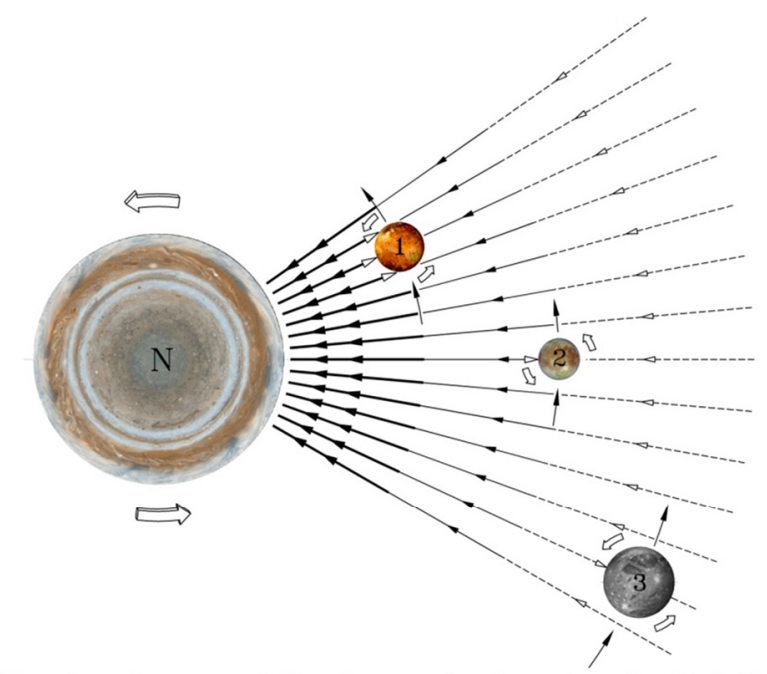

This schematic representation shows a view from above the North Pole of a Gas Giant and three "Synchronously rotating" major satellites. This diagram is not to scale. The gravitational pull of the Mother is represented by radiating lines, initially strongly solid and progressively spinning on their axes in a counter-clockwise direction, as indicated by spinning on their axes in a counter-clockwise direction, as indicated by intense, making it orbit the fastest, in a counter-clockwise direction around the Mother (solid arrow), and in the process encounters a stron drag on its leading edge. This directs the satellite to spin on its axis in a counter-clockwise fashion, thus augmenting its inherent tendency to spin in that same direction. The 2nd and 3rd satellites encounter less intense gravitational pull and, although still spinning synchronously, both the orbital period and axial rotation period are greater.

Figure 2. A gas giant and three of it's synchronously rotating major satellites

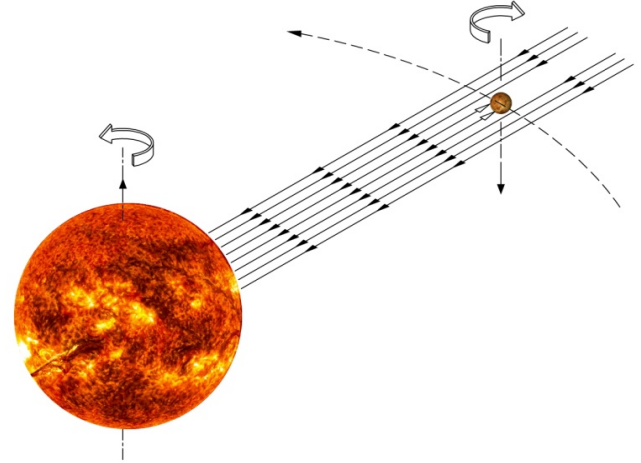

This schematic representation of the Sun and planet Venus is not to scale. The Sun's axial rotation is represented by the curved open arrow above the axis and it is counter-clockwise. The horizontal lines represent the gravitational pull of the Sun and the arrows on the lines indicate the direction of the pull. Venus is tilted 177.4 degrees (solid arrow pointing downwards) and its axial rotation is clockwise (i.e. it is spinning counter-clockwise, but its upside down orientation gives the illusion of "Negative" Rotation). Venus is still orbiting the Sun in a counter-clockwise direction and during this motion, it's leading edge encounters the tug of Sun's gravity. This Sun translates to an instruction to rotate in the counter-clockwise direction. This then is in conflict with Venus counter-clockwise direction. This then is in conflict with Venus delay so that Venus' axial rotation period becomes -5,832.5 Hrs.

Figure 3. Mechanism underlying the "Negative" rotation of the planet Venus and the inordinate delay

Shown in Figures 1a-1c are the relationships between the distance of the major (also closest) satellites from their respective gas giant central bodies and the speed of their (satellites') axial rotations. This finding has major implications in the phenomenon of synchronous rotation of these satellites, and bolsters the idea put forward in this paper that spin of the mother bodies has major impact on the behavior of their satellites, besides the effect on their orbital velocities. The mother bodies actually augment the speed of the axial rotation of the synchronously rotating satellites. The NASA's website did not provide similar details about the major (synchronously rotating) satellites of Neptune.

Figure 2 shows a schematic representation of a gas giant and three of its closest, synchronously rotating major satellites, with the hypothetical gravitational force-field emanating from the gas giant, and interacting with the corresponding gravitational waves of the satellite bodies. It depicts how the dominant gravitational pull from the mother planet both makes the satellites orbit faster, the closer they are to the mother and in the same direction as the mother's axial spin. It also illustrates how the same force fields from the mother tug on the leading edge of the satellites, thus making the satellites spin on their axes faster and in the same direction as the mother's axial rotation. This sequence of effects is behind the phenomenon of synchronicity; the closer the satellite, the faster the orbit and correspondingly faster the axial spin.

Table 4 compares the planets with the most pronounced axial tilts (Venus, Uranus and Pluto) with two "typical" planets, the Earth and Jupiter. The former three planets have 'negative' rotation (axial rotation opposite to the Sun's) and, the solid planets (Venus and Pluto) also have considerable delay in the rotation period. A reasonable inference from these observations is that, the increased axial tilt makes these planets' axial rotation compete with the gravitational/rotational influences from the mother (Sun) and thus, a considerable delay in their axial rotation occurs. The reason for lack of delay of the axial spin of Uranus is unknown but one plausible explanation may be that since it is a gas giant, despite its increased axial tilt, the axial rotation is not slowed, as its advancing edge 
may not offer the necessary grip or friction to experience the tug from Sun's gravitation. Or, perhaps, for the axial rotation to slow down, the tilt may have to be much higher, quite possibly higher than $100^{\circ}$.

Figure 3 explains how the excessive axial tilt $\left(-177.4^{\circ}\right)$ of Venus leads to the inordinate delay in its axial rotation and to 'negative rotation'. The dominant gravitational pull from the Sun, in concert with the Sun's axial rotation determines the orbital velocity of Venus. However, since Venus is essentially tilted upside down, and thus giving the spurious appearance of clockwise axial rotation, its advancing edge experiences a 'negative rotational influence' from the Sun, (instruction to spin in the same direction as the Sun's axial rotation, which is counter-clockwise). This leads to conflict and the considerable delay in Venus' axial rotation (-5832.5 hours) as well as the apparent "negative axial rotation".

\section{Statistical Analyses}

The statistical analysis associated with this research stems from the special relationships between the gas giants and their major satellites. Pearson's correlation coefficient (r) was calculated and the results are given below.

\section{Correlation Between the Planets and Their Moons}

In the statistical analysis, the independent variable, distance from the mother was compared to the dependent variable, the orbital period (or rotation period) and the correlation coefficients were calculated. The data values for the earth and three gas giants and their moons are shown in Table 3 and plotted in the associated Figures 1a $1 \mathrm{c}$.

When the data for the independent variables, orbital distance of the moons for the three gas giants, Jupiter, Saturn, and Uranus, were compared to the dependent variables, orbital period and then subjected to the correlation calculations, the correlation value of $r=0.9959,0.9893$, and 0.9962 respectively as indicated in Table 5. As Figures 1a-1c show this is an extremely strong positive correlation and indicates strong statistical support for the hypothesis that the variables of distance from the mother and rotation period (or orbital period) of the major satellites of these three Gas Giants are related.

Table 5. Correlation table for distance from mother body vs. Orbital parameters of satellites

\begin{tabular}{ll}
\hline Comparison & Corr. \\
\hline Distance from jupiter vs. Orbital period of the moons & 0.9959 \\
Distance from saturn vs. Orbital period of the moons & 0.9893 \\
Distance from uranus vs. Orbital period of the moons & 0.9962 \\
\hline
\end{tabular}

\section{Discussion}

\subsection{Increased Speed of Axial Rotation from Proximity to the Gas Giants in Synchronously Rotating Satellites}

Information in sufficient details on the satellites exhibiting synchronous rotation was available for the major satellites of Jupiter, Saturn and Uranus. The major finding presented in this paper is the influence on the speed of axial rotation of satellites due simply to proximity to the mother body. This is in addition to the speed of orbit of such satellite bodies, which are also directly proportionate to the distance from the mother body. While the latter is easy to understand, the former is harder to explain. The current author theorizes that the mother is able to enhance the speed of axial rotation of the satellite in the following way: The gravitational pull from the mother body is present all through and around the body, which keeps the satellite(s) tethered to the mother body. As the satellite orbits the mother, also due to the confluence of gravity and spin, the leading edge of the satellite experiences a tug towards the mother and it responds by increasing the speed of rotation, in the same direction as the mother's axial rotation. (Figure 2 illustrates this process). The closer the satellite, the less the axial tilt and the faster the orbit of the satellite and also its speed of axial rotation. The standard 'default' explanation in cosmological teaching, of 'tidal forces' cannot even begin to address this crucial finding. We propose a plausible explanation of why the augmented axial rotation of the satellite(s) might be a purposeful consequence and not just a by-product of the increased orbital speed, at a later section in the Discussion. How this same relationship between the mother's gravitation and direction of axial spin will impact the 'negatively' rotating satellites, in slowing the satellite's axial rotation is explained in the appropriate section below.

The findings reported in this paper and their interpretations explain the observed phenomena in the solar system better than Newton's or Einstein's ideas. Newtonians suggest that the planetary motion is due to the combined effect of gravity and his $1^{\text {st }}$ Law of motion. Einstein teaches that the satellites are situated at their locations due to a 'space-time warping' effect from the mother body (Einstein, 1920). Both of them cannot explain the direction 
of either the axial rotations of satellites or their orbits and the fact that almost always such orbital motions align with the mother bodies' axial rotation. Both of them also ignore the fact that all free-standing bodies rotate on their axes. Our interpretations of the observed phenomena explain completely why bodies remain in the orbital motions in perpetuity, while also imparting order to such movements. Hubble's finding of increased red-shift of light from distant galaxies and his interpretation that there was initially a "Big Bang" (Hubble, 1929) and, the subsequent teaching of an "expanding universe" (Smoot et al., 1992; Guth, 1981; Linde, 1982; Albrecht \& Steinhardt, 1982; Perlmutter et al., 1999; Reiss et al., 1998; Kirchner, 2003; Peebles \& Ratra, 2003; Bardeau, Steinhardt, \& Turner, 1983; Starobinskii, 1982; Hawking, 1982; Harrison, 1970; Guth \& Steinhardt, 1984) ignore all the axial rotational and orbital movements of the celestial bodies. Hubble's interpretations of a 'radial' motion of the galaxies is at odds with the finding of the orbital motions of all bodies up to the stage of the stars and even the stars' movements within the galaxies, which are all in a circumferential direction, but the galaxies themselves (which are, of course, made up of the stars and all the rest of the seen and unseen matter) as traveling in a radial direction. Common sense dictates that galaxies themselves will also move in a circumferential fashion. In the following sections, the current author explains in detail how spin and gravity can account for all the rotational and orbital movements, in not only the solar system bodies but also the larger universe.

\subsection{Role Played by Axial Tilt in "Synchronous" and "Negative" Rotations of Planets and Some of Their Satellites}

A scrutiny of the Tables 1,2 and 4 will reveal a curious relationship between the axial tilts of the satellite bodies and the presence of 'synchronous' rotation, nonsynchronous rotation or a 'negative' rotation of the satellites. In the case of the planets, (Tables 1 and 4) the three that have axial tilts larger than $90^{\circ}$ display 'reverse' or 'negative' rotation; these are Venus, Uranus and Pluto. This phenomenon means the direction of the axial rotation of these bodies is opposite to the direction of rotation of the parent and all the other bodies. The reverse is true for those bodies that have negligible tilts of their axes (less than $1^{\circ}$, Tables $2 \& 3$ ); the closest large satellites of Jupiter, Saturn, Uranus and Neptune are examples. Those bodies that have axial tilts between these extremes have 'normal' axial rotation. The question is why? It is believed that the explanation lies in the effect the mother bodies have on their satellites' axial rotation itself by the spin, such an effect emanating from the dominant body. As explained earlier, the closeness to the mother body makes those satellites orbit and spin on their axes faster and this latter effect leads to the axis of the satellites to become more aligned with the mother body's. This is just like a fast-spinning top versus a slow spinning top. Interestingly, this full alignment also leads to more thorough influence from the mother body and thus augmentation of the orbital speed and in its turn, increase of axial spin; thus, a mutually complementary arrangement obtains. In the case of the 'negative' rotation, since the axes of the bodies are tilted sufficiently, the net effect is that the mother bodies' influence, again through the combination of gravity and spin is opposite to that of the satellites'. Thus, a slowing of the satellite's axial rotation occurs, as well as a suggestion of rotation in the 'reverse' direction; it is as though the mother body is instructing the satellite to rotate in one direction but since the satellite's axis is tilted by so much, the satellite's own tendency competes with the mother's instruction and thus is slowed. As was stressed by us in a prior article (Physics Essays, 26:2, June 2013, Ref\# 19) this is a particularly telling phenomenon; it attests to the fundamental nature of the direction of rotation of the bodies. One could conclude that the normal axial rotation is counter-clockwise, relative to the North Pole, in all bodies and in all systems.

Earth's only moon displays the same synchronous rotation like the closest satellites of the gas giants. Situated at $384,000 \mathrm{Km}$ from the earth, the moon orbits in the same direction as the mother's axial rotation. Curiously, during all of its orbits, through the phases (which depend on the shadow cast by the earth during the moon's transit) an observer from the earth only sees one side of the moon. Now, how is that possible? Since the moon is also spinning on its own axis, while also orbiting the earth in a very close range, it behaves like the closer large moons of Jupiter, Saturn, Uranus and Neptune. The moon's axial rotation takes 27.322 earth days, while its orbital period is 27.3217 days. This is a very special relationship between the moon and the earth at present and it depends on the distance between the two bodies at this moment in time. As the moon is slowly moving away from the earth, this special relationship will change steadily. Solar eclipses will slowly change from being complete to one with a complete ring of sun showing around the moon, sometime in the future.

\subsection{Diminishing Orbital Velocity with Distance from Mother Bodies}

The diminishing orbital velocity of the satellites depending on the distance from the mother body clearly points to diminishing gravitational pull from the mother. However, gravity alone will not be sufficient to explain why the satellite bodies orbit. Newtonians' teaching that the combination of mutual gravitation and Newton's $1^{\text {st }}$ law of motion, cannot explain the direction of the orbits matching that of the mother body's axial spin, the orbital velocity diminishing depending only on the distance from the mother and all the satellites being situated around 
the ecliptic of the mother bodies. Einstein's "warping of the fabric" of "space-time" by the gravity of the celestial bodies will only help explain why other bodies might be situated where they are situated, but cannot explain why all bodies are constantly in motion, rotate on their own axes and orbit a larger neighbor at only around the ecliptic and with a diminishing orbital velocity, the farther away they are from the mother bodies.

The increase in orbital speed in satellites that are close to the mother bodies has the important consequence of increasing the centrifugal force to counter the increased gravitational pull. Otherwise, the close-by satellites will simply crash into the mother, rather than remain in orbit. The mother body controlling the orbital direction of the satellite bodies has the equally important function of maintaining an orderly system of orbits.

\subsection{The Interplay of Gravity and Spin in Other Situations}

Examples abound of the complementary interactions between mutual gravitation between celestial bodies and their axial rotations. This interplay of gravity and spin applies to the behavior of matter at the smallest, as well as that of the grandest. The effect on the matter at the grandest (planets, stars and galaxies) is to maintain their motion in space only in one direction and at specific velocities, both of which help maintain order in the universe; otherwise, if all bodies were to orbit at random the bodies will be constantly colliding with one another. There are several other phenomena in the universe that this combination can help explain. One is the shape that large bodies such as stars and planets assume. When such bodies form, the gravitational pull of matter that makes up these bodies, compresses this matter from every direction and the bodies assume a roughly spherical shape. However, all these bodies are not totally spherical; they bulge somewhat around the equator, making the circumference around the equator more than that through the poles. This is due to the centrifugal force exerted on matter by the axial spin of the body itself. In fact, this effect is what counter-balances the incessant inward pull of gravity in stars, rather than the usual belief that the nuclear fusion is responsible; otherwise, the shape assumed by stars might look more irregular. This idea is dramatically reinforced by the shape assumed by a star that rotates on its axis at an extremely high speed (the star is "VFTS 102" nick-named "Burger Star", (Dufton et al., 2011) which is located 160,000 light years away, in the Large Magellanic Cloud, a satellite galaxy of Milky Way Galaxy. It rotates on its axis at 1 million miles per hour!). The extreme degree of bulging at the equator displayed by this star is a testament to this shearing force imparted on the body at the equator, by the extreme rapidity of its axial spin. We are tempted to predict that this star is destined to explode into a supernova and give rise to a neutron star, in the future. Another good example of the complementary effect of the inward push of gravity and the counter-balancing effect of axial spin is the shape attained even by bodies that do not ignite, like the planets and their satellites, which also become almost spherical but with slight bulge in the equatorial regions.

Finally, we will explore certain anomalies in our solar system that seem to contradict the central theme of this paper. We propose that the first finding described below is an aberration and that most star systems display the expected pattern, that of synchronous rotations of closest planets and nonsynchronous, followed by negative rotations in the more and more distant planets, similar to the pattern the gas giants and their satellites follow in our solar system. The two planets that are closest to the sun, Mercury and Venus are both candidates for synchronous rotation. However, not only do they not rotate faster and thus rotate synchronously, their axial rotations are extremely slow. We think the slowness of the rotation in Mercury is due to the planet being composed mostly of iron. Thus, this planet behaves like an iron bar standing next to a bar magnet; the intense magnetic influence from the sun might be interfering with the axial rotation of Mercury and thus interfering in the effect from the gravitational forces. Venus suffers from the fact that its axis is tilted almost 180 degrees and, as explained in this paper, its axial rotation is inordinately delayed. Second special situation is that the gas giants seem to rotate very rapidly on their axes (Jupiter rotates once in $9.9 \mathrm{~h}$, Saturn in $10.7 \mathrm{~h}$, Uranus in $17.2 \mathrm{~h}$ and Neptune in 16.1h), which are much faster than that will be expected for such distant planets. The inference from the above is that the rotatory effect from the sun on these distant planets is significantly feebler than the planets' own intrinsic axial rotation rates. We assume this is due to the fact that the gaseous planets do not offer sufficient surface friction for the rotatory effect from sun to have any significant impact or control, on the 'advancing edge' of the planets. It may also be because the intrinsic axial rotation of the gas giants is so much faster that they far outpace any additional effects from the sun. In all instances, however, the orbits follow the dictates of the inverse square law; the inference is that the mutual gravitational influences on the planets from the sun and on the satellites from the gas giants, being felt on the whole bodies of the satellites and thus all satellite bodies behave similarly, as far as their orbits are concerned.

The rest of the planets in general display nonsynchronous rotation. It will be interesting to see if the most peripheral small ('dwarf') planets have increased axial tilts and are rotating negatively, just like Pluto. It will also be of interest to check other star systems and see if the "exo-planets" follow the general pattern that we report in 
our own gas giants and their satellites.

Across the universe there will be very many unusual findings. There may also be many star systems that have solid (terrestrial) planets without unusual features and thus behave similarly to the satellites of the gas giants in our solar system. Those exo-planets that have been noted to be Jupiter-sized but are situated close to the parent star and are orbiting the star extremely fast (Wang, Fischer, Elliott, \& Xu, 2015; Dawson \& Johnson, 2018) may be examples of synchronously rotating planets. Also, gaseous planets may abound in the universe and they also may rotate on their axes more rapidly, and independent of the central star.

Mention must be made of how large bodies such as planets and stars are able to rotate on their axes so fluidly. We believe this is due to the fact that all celestial bodies, where they are situated in the vast void of space, encounter no friction and thus they are able to display their own inherent tendency to spin on their axes. Further, since the distracting gravitational influences in those locations are also negligible and all bodies are essentially weightless. Precisely due to these circumstances, larger bodies from great distances are able to influence their satellite bodies through the confluence of gravity and spin, as explained previously. We do not know if the extreme cold of interstellar and interplanetary locations plays a role as well.

\section{Conclusion}

The finding of mother bodies' contribution to the speed and direction of the orbits and even the speed of the satellites' axial rotation offers compelling evidence for the importance of the fundamental property of matter to spin. In fact, not only does spin cooperate with gravity to impart order in the solar system, without this crucial property, it is hard to imagine the observed planetary and satellite motions. These effects can be summed up as follows: The inherent property of the mother bodies to rotate on their axes, coupled with mutual gravitation will 'grab' the satellite bodies and guide them to orbit in the same direction as its axial rotation. This paper illustrates how the distance from the mother determines both the speed of the orbits as well as the speed of axial rotation of the satellites. These two effects, with the aid of centrifugal force, keep the bodies in the appropriate locations and lead to the perpetual nature of the orbital movements. Indeed, an application of the manner of the intimate interaction between gravity and spin is sufficient to explain the origin of motion in the proto-stars and the planetary disk systems, and other areas in the universe such as the ring systems. It also explains why such motions remain in perpetuity. As galaxies are congregations of star systems, the same properties can be expected to drive these systems as well. It seems appropriate to assign a vital function for spin in the solar system and the universe, and not simply discount it as an incidental finding. The above explanations of the perpetual motions of celestial bodies necessarily contradict the scientific teaching of the past several centuries, that is the notion of a "conservation of angular momentum". If such a "conservation" is what leads to the presence and direction of axial rotation of celestial bodies, as the remnant of the rotation of the proto-planetary disks of solar system formation, what explains the rotation of matter beyond the star systems, the galaxies? Surely, a star's formation cannot impart a "conservation of its angular momentum" to the whole galaxy's circular motion in space.

\section{Acknowledgements}

The author is deeply indebted to Ms. Rosie Gonzales for her excellent secretarial assistance and to Mr. Chamel Raghu for help with the figures. Sincere gratitude is also due to Dr. Lloyd Taylor for the many helpful suggestions, for providing statistical analysis of the data and for the construction of Figures 1a-1c.

\section{Conflict of interests}

The authors declare that there is no conflict of interests regarding the publication of this paper.

\section{References}

Albrecht, A., \& Steinhardt, P. J. (1982). Cosmology for Grand Unified Theories with Radiatively Induced Symmetry Breaking. Physical Review Letters, 48(17), 1220-1223. https://doi.org/10.1103/PhysRevLett.48.1220

Bardeen, J. M., Steinhardt, P. J., \& Turner, M. S. (1983). Spontaneous creation of almost scale-free density perturbations in an inflationary universe. Physical Review D, 28(4), 679-693. https://doi.org/10.1103/PhysRevD.28.679

Dawson, R. I., \& Johnson, J. A. (2018). Origins of Hot Jupiters. Annual Review of Astronomy and Astrophysics, 56(1), 175-221. https://doi.org/10.1146/annurev-astro-081817-051853

Dufton, P. L., Dunstall, P. R., Evans, C. J., Brott, I., Cantiello, M., de Koter, A., ... \& Langer, N. (2011). The VLT-FLAMES Tarantula survey: The fastest rotating O-type star and shortest period LMC pulsar-remnants of a supernova disrupted binary?. The Astrophysical Journal Letters, 743(1), L22. 
Einstein, A. (1920). Relativity: The Special and General Theory. Random House, N.Y.

Gladman, B., Quinn, D.D., Nicholson, P., \& Rand, R. (1996). Synchronous Locking of Tidally Evolving Satellites. Icarus, 122(1), 166-192. https://doi.org/10.1006/icar.1996.0117

Guth, A. H. (1981). Inflationary universe: A possible solution to the horizon and flatness problems. Physical Review D, 23(2), 347-356. https://doi.org/10.1103/PhysRevD.23.347

Guth, A. H., \& Steinhardt, P. J. (1984). The Inflationary Universe. Scientific American, 250(5), 116-128. https://doi.org/10.1038/scientificamerican0584-116

Harrison, E. R. (1970). Fluctuations at the Threshold of Classical Cosmology. Physical Review D, 1(10), 2726-2730. https://doi.org/10.1103/PhysRevD.1.2726

Hawking, S. W. (1982). The development of irregularities in a single bubble inflationary universe. Physics Letters B, 115(4), 295-297. https://doi.org/10.1016/0370-2693(82)90373-2

Hubble, E. (1929). A RELATION BETWEEN DISTANCE AND RADIAL VELOCITY AMONG EXTRA-GALACTIC NEBULAE. Proceedings of the National Academy of Sciences of the United States of America, 15(3), 168-173. https://doi.org/10.1073/pnas.15.3.168

Kirshner, R. P. (2003). Throwing light on dark energy. Science (New York, N.Y.), 300(5627), 1914-1918. https://doi.org/10.1126/science.1086879

Linde, A. D. (1982). A new inflationary universe scenario: A possible solution of the horizon, flatness, homogeneity, isotropy and primordial monopole problems. Physics Letters B, 108(6), 389-393. https://doi.org/10.1016/0370-2693(82)91219-9

Murray, C. D., \& Dermott, S. F. (2012). Solar System Dynamics: Cambridge University Press. https://doi.org/10.1017/CBO9781139174817

Nakamura, T. (1989). Binary Sub-Millisecond Pulsar and Rotating Core Collapse Model for SN1987A. Progress of Theoretical Physics, 81(5), 1006-1020. https://doi.org/10.1143/PTP.81.1006

Peebles, P. J. E., \& Ratra, B. (2003). The cosmological constant and dark energy. Reviews of modern physics, 75(2), 559.

Perlmutter, S., Aldering, G., Goldhaber, G., Knop, R. A., Nugent, P., Castro, P. G., ... \& Hook, I. M. (1999). Measurements of $\Omega$ and $\Lambda$ from 42 high-redshift supernovae. The Astrophysical Journal, 517(2), 565.Seeds, M. A. (1999). Inverse Square Law, Stars and Galaxies, 82-3. Wadsworth, Belmont, CA.

Raghuprasad, P. K. (2013). Planetary spin-orbit attributes in the solar system and their wider implications. Physics Essays, 26(2), 331-338. https://doi.org/10.4006/0836-1398-26.2.331

Riess, A. G., Filippenko, A. V., Challis, P., Clocchiatti, A., Diercks, A., Garnavich, P. M., . . Tonry, J. (1998). Observational Evidence from Supernovae for an Accelerating Universe and a Cosmological Constant. The Astronomical Journal, 116(3), 1009-1038. https://doi.org/10.1086/300499

Smoot, G. F., Bennett, C. L., Kogut, A., Wright, E. L., Aymon, J., Boggess, N. W., ... \& Hinshaw, G. (1992). Structure in the COBE differential microwave radiometer first-year maps. The Astrophysical Journal, 396, L1-L5.Starobinskii, A. A. (1982). Lett., B117, 175-178.

Wang, J., Fischer, D. A., Horch, E. P., \& Huang, X. (2015). ON THE OCCURRENCE RATE OF HOT JUPITERS IN DIFFERENT STELLAR ENVIRONMENTS. The Astrophysical Journal, 799(2), 229. https://doi.org/10.1088/0004-637X/799/2/229

\section{Copyrights}

Copyright for this article is retained by the author(s), with first publication rights granted to the journal.

This is an open-access article distributed under the terms and conditions of the Creative Commons Attribution license (http://creativecommons.org/licenses/by/4.0/). 